\title{
Design of a Plasmonic Platform to Improve the SERS Sensitivity for Molecular Detection
}

\author{
Chahinez DAB ${ }^{*}$, Reji THOMAS ${ }^{2}$, and Andreas RUEDIGER ${ }^{3}$ \\ ${ }^{1}$ Chemistry Department, Université de Montréal, Montreal H3C 3J7, Canada \\ ${ }^{2}$ Division of Research and development, Lovely Professional University, Punjab 144411, India \\ ${ }^{3}$ Nanophotonics-Nanoeletronicss, Institut National de la Recherche Scientifique INRS-EMT, Varennes J3X 1S2, Canada
}

${ }^{*}$ Corresponding author: Chahinez DAB_ E-mail: chahinez.dab@umontreal.ca

\begin{abstract}
We suggested a plasmonic platform based on a cubic pattern of gold spheres for surface enhanced Raman spectroscopy (SERS). In the case of linear polarization along the symmetry axes, the SERS enhancement per area is identical to hexagonally patterned surfaces. The validity of this model was tested using the simulation package of COMSOL Multiphysics ${ }^{\circledR}$ Modeling Software. We found an improved sensitivity in the near infrared and visible region of the electromagnetic spectrum. This method considered tolerance towards stacking faults and suggested a plasmonic platform for ultra-sensing applications. The design can be extended towards the molecular detection if the proposed plasmonic platform is used with SERS.
\end{abstract}

Keywords: Plasmonic platform; SERS enhancement; sensitivity; finite element method

Citation: Chahinez DAB, Reji THOMAS, and Andreas RUEDIGER, "Design of a Plasmonic Platform to Improve the SERS Sensitivity for Molecular Detection," Photonic Sensors, 2020, 10(3): 204-214.

\section{Introduction}

Surface enhanced Raman spectroscopy (SERS) depends on plasmonic platforms and nano-antenna which have evolved into an active field of research [1-3]. The design and fabrication of conductive nanostructures for the control and manipulation of the confined electromagnetic optical field are the main activities in this field. The resulting resonance frequencies of those nanostructures span a spectral range from the ultraviolet to the near infrared depending on the dimension of such structures. For a plasmonic platform, even multiple resonances due to plasmonic modes might occur depending on the excitation wavelength. The optical signature of those nanostructures is determined by the localized surface plasmon resonance (LSPR) confined in close proximity of the apexes of the nanostructures. LSPR is very sensitive to different factors, such as the refractive index of the medium, the size as well as the material used [4-6]. On SERS, the local enhancement is maximized when the LSPR frequency of the plasmonic platform matches the resonance frequency of the excitation light. This is particularity exploited in SERS in order to increase the sensitivity and to enable single molecule detection [7-9]. In general, the Raman signature of molecules placed on the plasmonic structure is amplified by several orders of magnitude by positioning them in the nanogaps of the plasmonic

Received: 13 June 2019 / Revised: 06 November 2019

(C) The Author(s) 2019. This article is published with open access at Springerlink.com DOI: 10.1007/s13320-019-0576-3

Article type: Regular 
nanostructures. So, to improve the sensitivity, the design of the nanostructures plays a crucial part as practical antennas are made from these results. This relates to the enhancement provided by each nanogap as well as the area density of these gaps. Other fields that can also benefit from plasmonic enhancement are sensors, nano photonics, biology, and medicine [10-14].

Gold and silver are the most suitable materials used for plasmonic platforms as both offer intense field enhancement due to a high density of quasi-free electrons and low losses in the relevant spectral range of interest from the visible to the near infrared $[15,16]$. Among many reported plasmonic platforms, a quartet of gold nanorods was developed by J. Kumar et al. [17], who found that a high electric field prevailing at the quartet junctions resulted in a large enhancement in the Raman signals of molecules. Similarly, A. Garreau et al. [18] developed a plasmonic platform based on a heterogeneous gold and silver nanotriangles, which is the most commonly used design of the plasmonic platforms. This platform enhances the near-field fluorescence in the proximity of nanotriangles as amplification occurs at the three apexes of the nanotriangle. In terms of the surface density of hot-spots, hexagonal patters with three-fold symmetry provide the best yield, which is however impossible to ideally excite more than one third of this population under linear polarization. Given the strong non-linearity of (1), the remaining two thirds of the nanogaps will be illuminated under 60 degrees polarization rotation, making their contribution to the overall SERS signal negligible.

In this study, we presented a novel design for a plasmonic platform to improve the percentage of excited hotspots, at the expense of area density. The design considers gold nanostars rather than nanorods or nanotriangles: two approaches are therefore considered and discussed as follows. In the first case, small plasmonic nanostars (SPNs) are designed with an edge radius of $2 \mathrm{~nm}$ on a glass substrate. One efficient way to fabricate those structures practically is by nanosphere lithography $[18,19]$ that will be discussed later, even though we acknowledge that the formation of hexagonally patterned structures is more straight-forward. In the second case, a plasmonic platform composed of large plasmonic nanostars (LPNs) with $20 \mathrm{~nm}$ radius of the edge is deposited above the SPNs. By combining SPNs and LPNs, we designed the whole platform and we were able to simulate the electromagnetic field confinement in close proximity of the nanostars. SERS intensity enhancement was then compared with its conventional plasmonic platform counterpart used without SPNs and a proof of concept of the sensitivity improvement from the visible to the near infrared was demonstrated and discussed.

\section{Methods}

The simulation was based on the finite element methods (FEM) with numerically stable edge element discretization and solutions of Maxwell's equations using COMSOL Multiphysics software. This classical electromagnetic approach discarded contributions from chemical enhancement in surface enhanced Raman spectroscopy or quantum mechanical effects due to conductive coupling between plasmonic features. The dependence of the relative permittivity $\varepsilon_{r}$ of gold on the frequency is introduced by (1)

$$
\varepsilon_{r}(\omega)=[n(\omega)-\mathrm{i} k(\omega)]^{2}=\mathcal{E}^{\prime}(\omega)-\mathrm{i} \varepsilon^{\prime \prime}(\omega)
$$

where $n$ and $k$ are real numbers, defining the refractive index and the extinction coefficient, respectively and $\omega$ denotes the excitation frequency of the incident light. $\varepsilon^{\prime}$ and $\varepsilon^{\prime \prime}$ are the real and imaginary parts of the relative permittivity, respectively. The dimensional space or spatial resolution is discretized using a non-uniform mesh method with a unit cell size of $1 \times 1 \mathrm{~nm}^{2}$ within the nanostars gap.

The plasmonic effect contributes dominantly to the enhancement of the electromagnetic field in 
SERS. In particular, the amplification of the electric field is pronounced in the so called "hot-spot" that is confined to the nanoscale gaps of the nanostructure geometry. This creation of "hot-spot" is mainly due to the formation of dimers. The enhancement effect occurs therefore in two fundamental steps: first, around the plasmonic nanoparticle, the local field is further enhanced and a dipole is induced leading to the enhancement of the Raman scattering from the molecule in the nanogap. This enhancement is described according to (2):

$$
G_{1}=\left[E_{\mathrm{loc}}\left(\omega_{0}\right) / E_{0}\left(\omega_{0}\right)\right]^{2}
$$

where $E_{\mathrm{loc}}$ and $E_{0}$ are the local electric fields in the presence and absence of nanoparticle, respectively. $\omega_{0}$ is the frequency of the excitation laser. We set the incident field $E_{0}$ to unity ( $E_{0}=$ $1 \mathrm{~V} / \mathrm{m})$. Then, in the second step, a mutual excitation from the system of the nanoparticles at a resonant frequency $\omega_{r}$ induces an enhanced apparent Raman polarizability according to (3):

$$
G_{2}=\left[E_{\mathrm{loc}}\left(\omega_{r}\right) / E_{0}\left(\omega_{r}\right)\right]^{2} .
$$

As a result, the enhanced Raman scattered light from the nanoparticles ( $G$ enhancement factor) is presented in (4) [20]:

$$
\begin{aligned}
G & =G_{1}\left(\omega_{0}\right) G_{2}\left(\omega_{r}\right)=\frac{\left|E_{\mathrm{loc}}\left(\omega_{0}\right)\right|^{2}\left|E_{\mathrm{loc}}\left(\omega_{r}\right)\right|^{2}}{\left|E_{0}\left(\omega_{0}\right)\right|^{2}\left|E_{0}\left(\omega_{r}\right)\right|^{2}} \\
& \approx \frac{\left|E_{\mathrm{loc}}\left(\omega_{0}\right)\right|^{4}}{\left|E_{0}\left(\omega_{0}\right)\right|^{4}} .
\end{aligned}
$$

This approximation only holds true for small Raman shifts for which the electromagnetic field enhancement of the excitation and signal wavelengths are having the same enhancement factor. The SERS enhancement factor $G$ thus depends on the gap size between the nanostars antennas; it increases as the distance of separation is reduced. Basically, there is a critical gap size, below which the enhancement factor is limited (for a sub-nanometer scale where quantum tunneling dissipates field energy, which is not considered in the current study).
The plasmonic platform used for the simulation is shown in Fig. 1(a). It consists of gold LPNs with $20 \mathrm{~nm}$ radius dimensions with a distance $d_{\text {LPNS }}$ between the apexes of LPNs and gold SPNs with 2 $\mathrm{nm}$ radius with a distance $d_{\mathrm{SPN}}$ between the apexes of SPNs, and both plasmonic nanostars are realized on a glass substrate. The overall LPNs gap is therefore filled with SPNs. To get a clear picture of the structure, LPNs alone (top) and LPNs with SPNs (bottom) are depicted in Fig. 1(b). Simulations have been performed on these structures in air as well in liquid to study the effect of surrounding medium on the plasmon resonance and field enhancements.

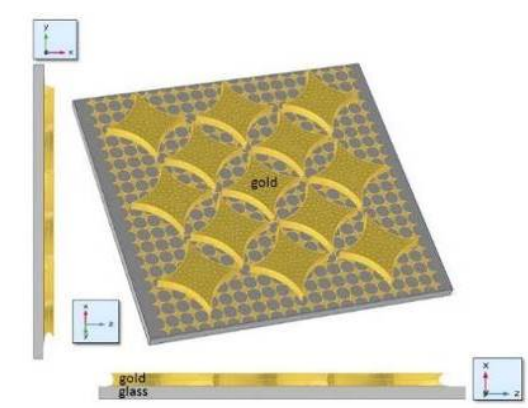

(a)
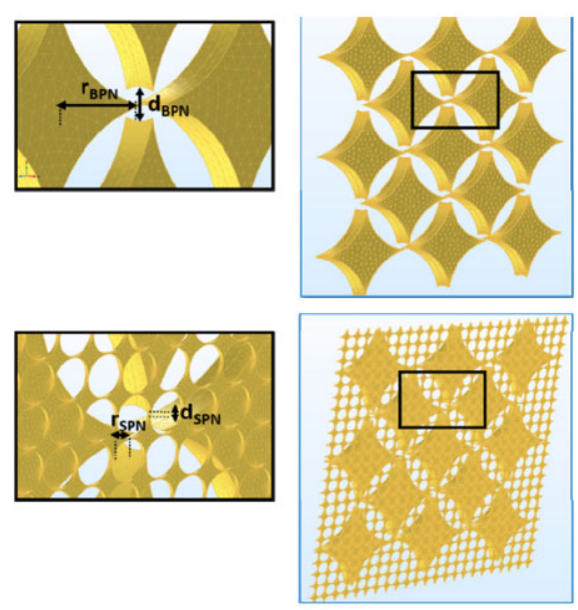

(b)

Fig. 1 Model of the plasmonic platforms: (a) design of the plasmonic nanostars platform LPNs: $20 \mathrm{~nm}$ radius $\left(r_{\mathrm{SPNs}}=2 \mathrm{~nm}\right.$ and $r_{\mathrm{LPNs}}=20 \mathrm{~nm}$ ) and (b) top left is the zoom of LPNS from the black rectangle on the fight and the bottom left is the zoom of the (SPNs+LPNs) from the marked area on the right.

\section{Results and discussion}

Absorption of the aforementioned plasmonic 
platform is studied as a function of excitation wavelength to visualize the plasmon mode existing for the two different configurations, LPNs and (SPNs+LPNs). We also investigated the effect of refractive index variations of the surrounding medium on the plasmon resonance.

\subsection{Absorption}

First, we investigated the effect of the distance between the nanostars $\left(d_{\mathrm{LPNs}}\right)$ on the absorption spectrum in order to identify the coexisting plasmons modes in the normal configuration (without SPNs). Figure 2 shows the normalized absorption cross section as a function of the wavelength from ultraviolet to near infrared spectrum $(200 \mathrm{~nm}$ to $900 \mathrm{~nm})$ for different distances between the apex of the nanostars $\left(d_{\mathrm{LPNs}}=1 \mathrm{~nm}, 5 \mathrm{~nm}\right.$, $7 \mathrm{~nm}$, and $10 \mathrm{~nm}$ ). As the distance increases, the number of plasmonic modes is decreased and a decrease in the absorption is also detected. Three plasmon modes are observed for $1 \mathrm{~nm}$ and $5 \mathrm{~nm}$. However, only two plasmonic modes are detected for $7 \mathrm{~nm}$ and $10 \mathrm{~nm}$ distances in the visible and near infrared NIR regions. The visible plasmonic mode $(550 \mathrm{~nm}-700 \mathrm{~nm})$ is shifted to higher wavelengths as the distance decreases as expected through an increased coupling and becomes narrower. This narrowing drastically improves the spectral resolution of that particular mode. The near-infrared plasmon mode is splitting into two plasmonic modes as the distance decreases $\left(\lambda_{\max 1}=750 \mathrm{~nm}\right.$ and $\lambda_{\max 2}=850 \mathrm{~nm}$ ). This is an indication that the behavior of the whole plasmonic platforms (quadrupole modes) nanostars is much more complex and differs from the behavior of a conventional dipole mode. The distance decrease leads to the shift of the mode to the higher wavelengths rather than splitting in the case of dipole mode between two metallic nanostructures [4, 21]. The shift of the plasmonic modes in the near infrared observed in the present nanostars platform is similar to the case of two coupled metallic nanoparticles approaching quantum regime [22, 23]. So, we can assign the wavelength red shift of the visible mode to the possible overlapping of the platform nanostars peaks for small distances.

Now, we fix a distance between the apex of the plasmonic nanostars to $5 \mathrm{~nm}\left(d_{\mathrm{LPNs}}=5 \mathrm{~nm}\right)$ and realize SPNs of $2 \mathrm{~nm}$ radius with LPNs as depicted in Fig. 1(b). SPNs in particular enhances the number of hotspots. While a top-down approach of lithography would always be a viable option to create these patterns, who are in principle achievable with stencil or bottom-up techniques [24-26].

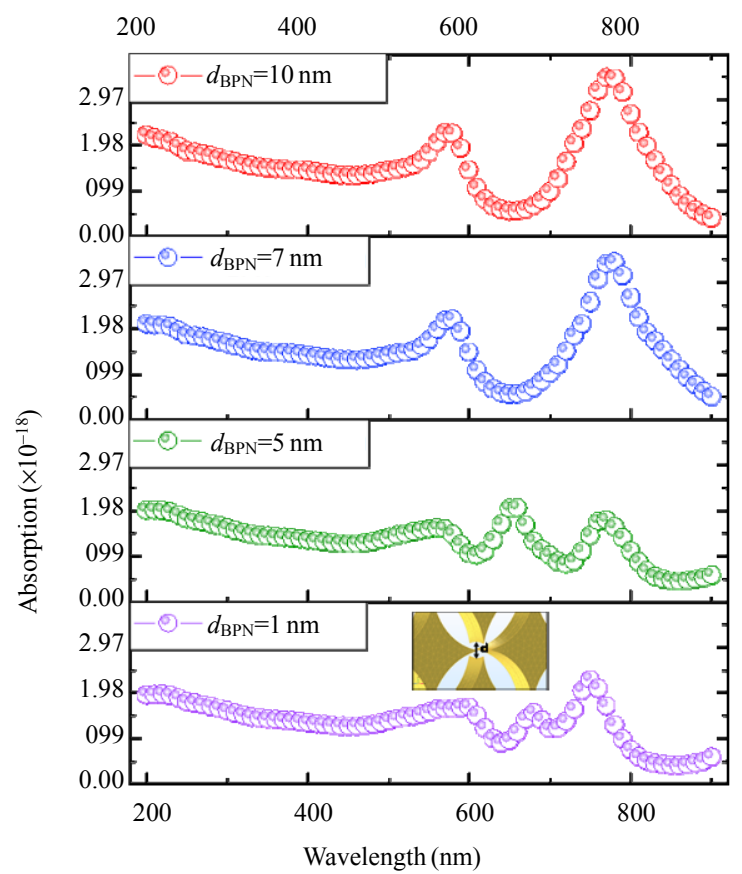

Fig. 2 Absorption of the plasmonic platforms without SPNs as a function of the wavelength variation for different distances between the apexes of the nanostars in air environment $\left(r_{\mathrm{LPNS}}=\right.$ $20 \mathrm{~nm})$.

The main advantage of stencil techniques is the low-cost fabrication of large arrays of metallic nanostructures with controlled size, shape, and interparticle distance. Recently, we used this technique in the fabrication of a hexagonal array of plasmonic gold nanotriangles on the glass substrate with unique dimension [27], which led to an efficient study of the localized surface plasmon 
resonance (LSPR) of the nanotriangles due to the spatial confinement of the electric field. Stencil techniques may be used as the most suitable for the fabrication of our proposed complex plasmonic platform composed of SPNs and LPNs as compared with self-organized techniques, given the preferential close-packed nature of hexagonal patterns. This study now addresses the quantification of the SERS effect and the LSPR modes, theoretically rather than experimentally. Figure 3 presents the absorption cross sections for the plasmonic platform with SPNs for different distances $d_{\mathrm{SPNs}}(1 \mathrm{~nm}, 0.7 \mathrm{~nm}$, and $0.5 \mathrm{~nm})$ between the small plasmonic nanostars. It should be noted that $0.3 \mathrm{~nm}$ presents the smallest physically meaningful gap distance that corresponds approximately to the bond length between two gold atoms which is $288.9 \mathrm{pm}[4,28]$. Therefore, in this study, we are limited to $0.5 \mathrm{~nm}$ distance between SPNs where the optical responses still obey Maxwell's theory (plasmon quantum limit exposed). A similar effect in the SPNs absorption spectrum is found when the distance between SPNs ( $\left.d_{\mathrm{SPNS}}\right)$ increases as observed for LPNs. We remark that there is a splitting of the near-infrared plasmon mode that redshifts the plasmon band to higher wavelengths. For the visible range, we detect two plasmon modes for $1 \mathrm{~nm}$ and $0.5 \mathrm{~nm}$ distances however only one plasmonic mode is detected for distance of $0.7 \mathrm{~nm}$, suggesting no trend in the appearance and disappearance of modes with distance $d_{\text {SPNs. }}$. This is an indication that the plasmonic platform with (SPNs+LPNs) behaves differently with respect to the LPNs platforms alone due to the mixing on the LPNs modes and SPNs modes. The hybridized modes of (SPNs+LPNs) makes the newly designed platform interesting for the detection of molecules. In addition to the hybrid mode behavior, the sensitivity of the near infrared mode increases as well.

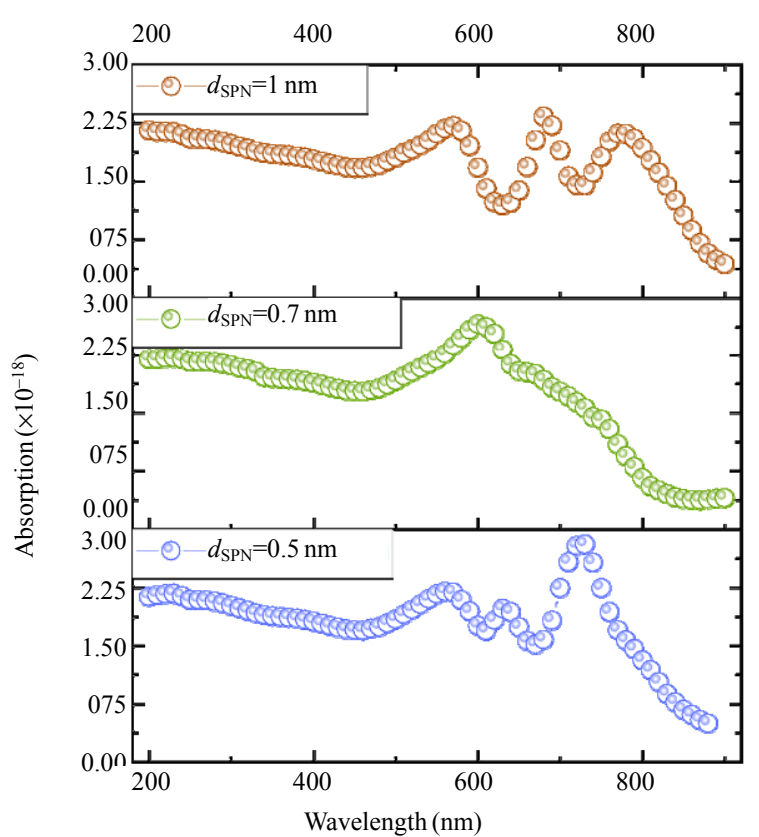

Fig. 3 Absorption of SPNs as a function of the wavelength variation with SPNs for different distances between SPNs in air environment.

\subsection{Sensitivity of the plasmonic resonance on refractive index}

In this section, we discussed the sensitivity that corresponds to the plasmonic mode with the highest absorption of the resonance wavelength as a function of the ambient refractive index. This feature is worth highlighting as it relates to the versatility of the platform for different solvents. We define the SERS sensitivity factor of the plasmonic platform as the ratio between the relative peak position $\Delta \lambda_{\max }$ in (nm) unit and the refractive index change $n$ expressed as refractive index unit (RIU). It was reported that the LSPR is sensitive to the refractive index of the medium and therefore red shift as the refractive index increases [29, 30]. The position of the peak shift depends on the real part of the permittivity, and in resonance, the SERS intensity is maximized due to the relation $[31,32]$ in (5)

$$
\varepsilon^{\prime}=-2 n_{\text {sol }}^{2}
$$

where $n_{\text {sol }}$ is the refractive index of the solvent.

To determine the performance of nanostars platform for practical sensing applications, we investigated changes in its plasmonic response when 
the refractive index $(n)$ of the surrounding medium is changed. Immersing the SPNs and LPNs platforms in various liquids with different refractive indices, we simulated the absorption spectrum as a function of refractive index for an excitation wavelengths varying between $200 \mathrm{~nm}$ and $900 \mathrm{~nm}$. To this end, we used the following liquids: methanol $\left(\mathrm{CH}_{3} \mathrm{OH}\right) n=1.3$, heptane $\left(\mathrm{C}_{7} \mathrm{H}_{16}\right) n=1.39$, Ethylene glycol $\left(\mathrm{C}_{2} \mathrm{H}_{6} \mathrm{O}_{2}\right) \quad n=1.43$, and glycerine $\left(\mathrm{C}_{3} \mathrm{H}_{8} \mathrm{O}_{3}\right)$ $n=1.47$. Figure 4(a) exhibits the computed absorption cross-section of the plasmonic platform nanostars with and without SPNs for different media with varied refractive index. The figure shows the presence of two visible modes; first mode between $500 \mathrm{~nm}$ and $600 \mathrm{~nm}$, and the second one between $600 \mathrm{~nm}$ and $700 \mathrm{~nm}$. The near-IR mode is observed between $750 \mathrm{~nm}$ and $900 \mathrm{~nm}$. Increasing in the refractive index of the liquids, redshifts the plasmonic modes of the visible and near infrared. This shift to longer wavelength is observed for platforms with and without SPNs. Resonances at visible wavelengths have been a great advantage for earlier studies [33-35]. However, adapting plasmonic platforms to applications that require operation at longer wavelengths has been a challenge. The integration of $\mathrm{Au}$ nanostars to biomedical applications requires resonances in the near infrared region as the reduced attenuation of light through biological tissues at these wavelengths improves the penetration depth [36, 37]. Herein, we showed that at least small plasmonic nanostars SPNs is an efficient way and contributes to the redshifts of the plasmonic band positions to the infrared spectrum, and thus is a promising solution to adopt for those applications.

More details on the refractive index of liquid environment effect on the sensitivity are summarized in Fig.4(b) that shows the sensitivity of the different modes. This figure suggests a linear dependence of the sensitivity with refractive index for all plasmon modes over the investigated wavelength range. For the near-infrared active mode, the curve has a large slope that presents the sensitivity factor $\left(\Delta \lambda_{\max } / \Delta n\right)$ increasing from $373 \mathrm{~nm}$ per RIU to $420 \mathrm{~nm}$ per RIU with the use of SPN. A similar improvement was found for the visible mode 1 with a sensitivity raise with SPN from $118 \mathrm{~nm}$ per RIU to $161 \mathrm{~nm}$ per RIU. However, the sensitivity of the visible mode 2 of the plasmonic platform with SPN was decreased from $213 \mathrm{~nm}$ per RIU to $140 \mathrm{~nm}$ per RIU. We conclude that (SPNs+LPNs) is the most sensitive and the best candidate for the SERS plasmonic platform.

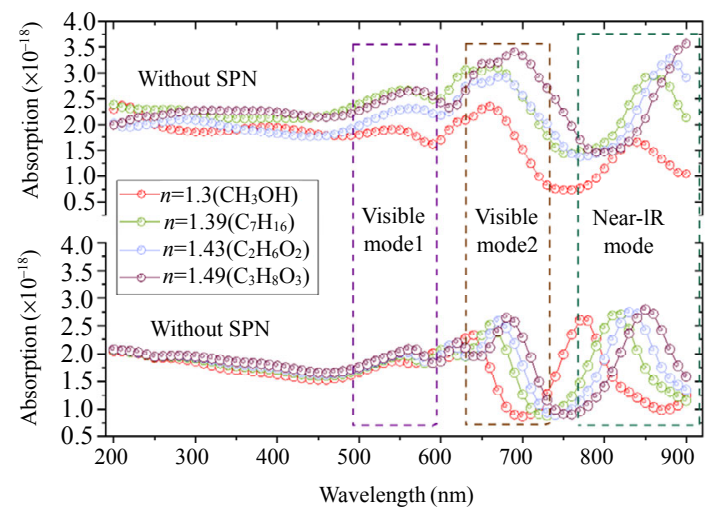

(a)

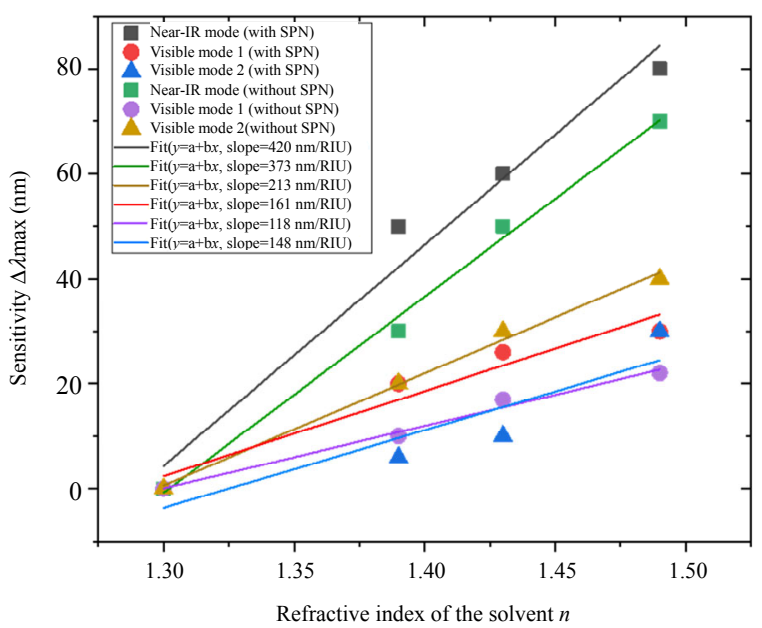

(b)

Fig. 4 SERS sensitivity of the plasmonic platform: (a) absorption as a function of wavelength for the plasmonic platforms nanostars with SPNs (curve on the top) and without SPNs (curve on the bottom) immersed in different liquids and (b) variation of the sensitivity (maximum peak position) for visible modes and near-IR mode in the plasmonic platform with and without SPNs as a function of the refractive index of the liquid $\left(r_{\mathrm{SPNs}}=2 \mathrm{~nm}, r_{\mathrm{LPNs}}=20 \mathrm{~nm}\right)$. 


\subsection{SERS intensity}

As the plasmonic gold nanostructures overlap, there is an enhanced field near the apexes that became intense and hot spot is created [38-40]. In term of SERS intensity, we excited the plasmonic platforms with and without SPNs with different laser wavelengths $(230 \mathrm{~nm}, 530 \mathrm{~nm}, 730 \mathrm{~nm}$, and $860 \mathrm{~nm})$ in air as medium. Figure 5(a) shows the enhancement factor (logarithmic scale) for a plasmonic platform without SPNs. All the cartographies indicate a hot spot localization at the vicinity of the apex of the gold nanostars that increases in terms of intensity as the excitation wavelength increases (red is very intense and blue is a very low field enhancement). Figure 5(b) depicts the SERS intensity on the cross section of one LPNs (black line). SERS intensity enhancement remains constant at 1.2 for wavelength between $250 \mathrm{~nm}$ and $450 \mathrm{~nm}$. Then it monotonically increases until it reaches 3.5 at $650 \mathrm{~nm}$. Between $650 \mathrm{~nm}$ and $745 \mathrm{~nm}$, SERS intensity enhancement drops again under unity. Beyond $750 \mathrm{~nm}$ wavelength, it again increases.
Figure 5(c) illustrates the SERS intensity enhancement on the cross section of the entire plasmonic platform (black line). In this case, the SERS intensity enhancement monotonically increases from $200 \mathrm{~nm}$ to $700 \mathrm{~nm}$ wavelengths. It reaches a value around 3 and then drops again. However, this configuration is not useful for the detection of molecules in the nanogap between the LPNs since it possesses a wider regions and the spatial resolution is up to $20 \mathrm{~nm}$. To overcome this issue, we present the SERS intensity when using our proposed SPNs with dimensions of $2 \mathrm{~nm}\left(r_{\mathrm{SPNs}}=2 \mathrm{~nm}\right)$ and a distance $d_{\mathrm{SPNs}}=1 \mathrm{~nm}$ in the gap between the LPNs. Figure 6(a) presents the enhancement factor (logarithmic scale) for the plasmonic platform with SPNs for different excitation wavelengths. Those SPNs are useful as they increase the electromagnetic field confinement between the LPNs and the spatial resolution, which is expected to be up to $2 \mathrm{~nm}$. The cartographies show a hot spot localization between the SPNs, the SPNs and the edges of LPNs, and between the LPNs as in the first case [Fig. 5(a)].
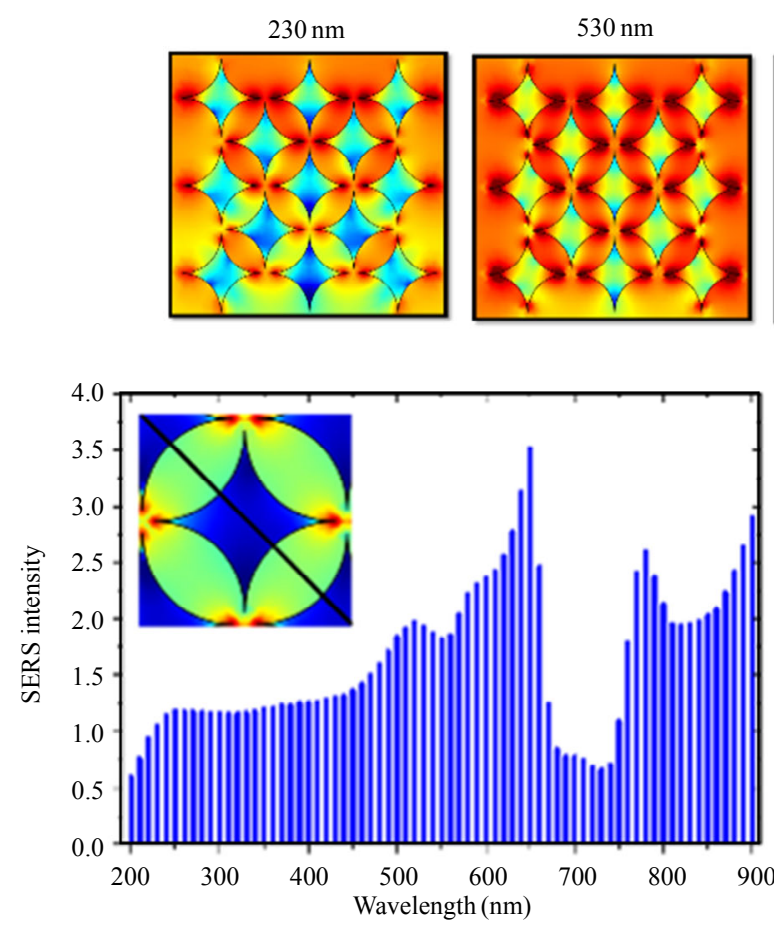

(b)

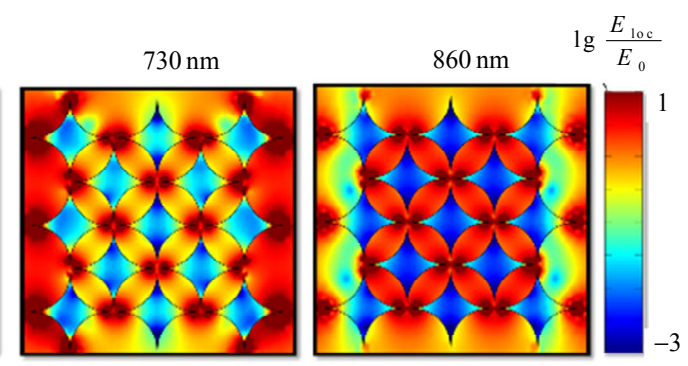

(a)

Fig. 5 Simulations of the plasmonic platforms: (a) SERS enhancement factor (logarithmic scale) for the plasmonic nanostrars platform without SPNs for different excitation wavelengths. SERS intensity cross section as a function of the excitation wavelength for (b) one LPNs, and (c) all plasmonic platforms of LPNs. 

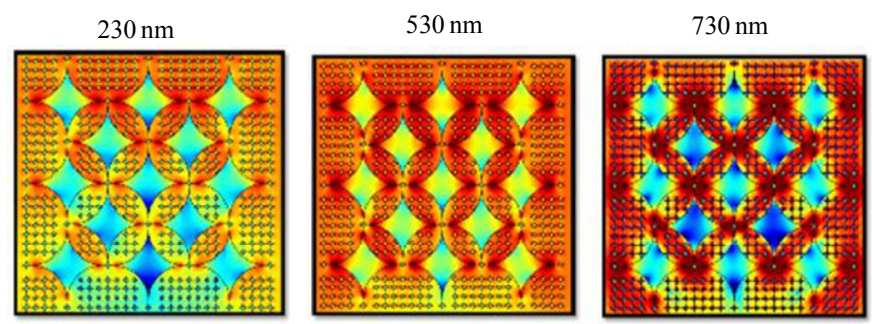

(a)

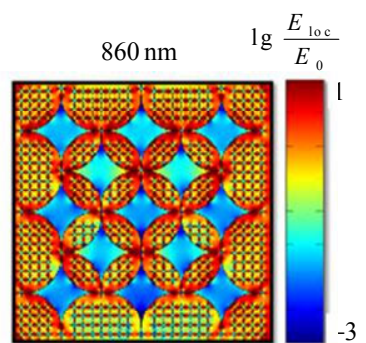

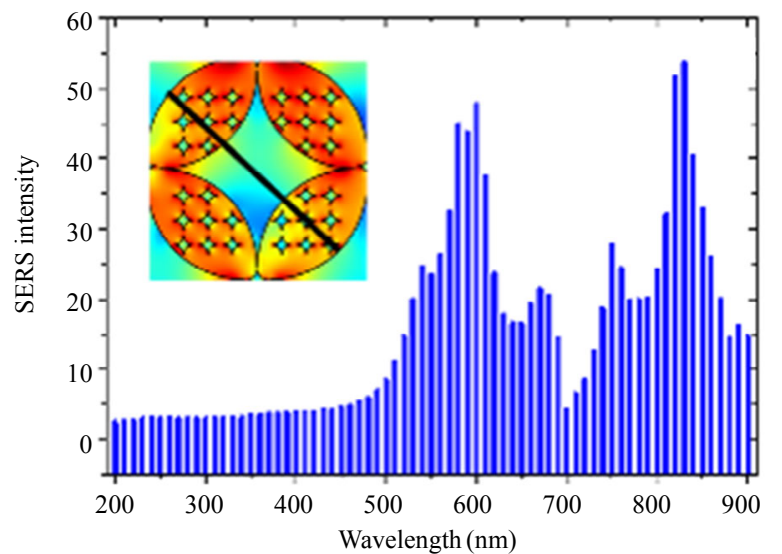

(b)

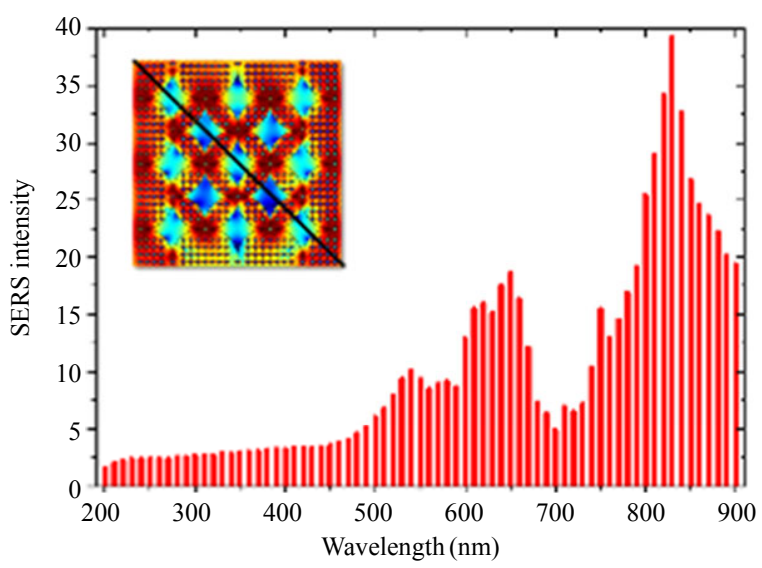

(c)

Fig. 6 Simulations of the plasmonic platforms: (a) SERS enhancement factor (logarithmic scale) for the plasmonic nanostars platform with SPNs for different excitation wavelengths. SERS intensity cross section as a function of the excitation wavelength for (b) one LPNs and SPNs, and (c) the all plasmonic platforms of LPNs and SPNs.

Those findings indicate that SPNs adds their enhancement effect on the platform and improves the SERS intensity on the surface of the platform. Figure 6(b) presents the SERS intensity one LPNs surrounded by SPNs on the edges and the apexes. As the excitation wavelength increases from $200 \mathrm{~nm}$ to $500 \mathrm{~nm}$, the SERS intensity remains constant at an enhancement factor of approximately 7. Then it increases between $500 \mathrm{~nm}$ and $600 \mathrm{~nm}$ with a maximum on intensity equal to 48 for $580 \mathrm{~nm}$ wavelength, 13 times improved with respect to a platform without SPNs. SERS intensity enhancement then decreases for an excitation wavelength between $600 \mathrm{~nm}$ and $700 \mathrm{~nm}$. Then it presents another enhancement maximum of 55 in the near infrared $(840 \mathrm{~nm})$. Surprisingly, the SERS enhancement in the near infrared regions (mode between $800 \mathrm{~nm}$ and $900 \mathrm{~nm}$ ) is not present for platforms without SPN [Fig. 5(c)], therefore SPNs contributes to the appearance and enhancement of this mode. This is the case over the entire platform surface as depicted in Fig. 6(c) that presents the SERS intensity improvement on a cross section of all the plasmonic platforms (black line), where the intensity is ameliorated by $85 \%$ between $750 \mathrm{~nm}$ and $900 \mathrm{~nm}$ excitation wavelength with respect to a platform without SPNs. The SERS intensity improvement and the increased number of hotspots by using SPNs should lead to an improved probability of molecules to experience the localized enhanced field and can therefore be detected with their SERS signature.

The direct comparison of hexagonal and cubic two-dimensional (2D) patterns for excitation with linearly polarized light with the polarization direction along one of the in-plane symmetry axes suggests that in a $2 \mathrm{D}$ hexagonal pattern, only $1 / 3$ of all gaps will yield substantial field enhancement 
which translates into a negligible SERS signal from the remaining 2/3 following the $G^{4}$ dependence of (1). For a cubic $2 \mathrm{D}$ pattern, half of all gaps will contribute to the overall SERS signal. This consideration has to be weighted with the area density of hotspots for the respective structures that is $50 \%$ higher for the hexagonal $2 \mathrm{D}$ pattern so that overall, both structures (hexagonal and cubic BNP) should provide identical overall SERS enhancement for the polarization along one of the symmetry axes. Other comparisons between the polygon shaped assemblies (digon, trigon, tetragon, pentagon, and hexagon) were performed by F. S. Ou et al. [41] where plasmonic nanostructures were excited with $785 \mathrm{~nm}$ laser. Authors found that all substrates generated qualitatively the same sets of Raman peaks with the similar peak intensity. However, pentagonal assembly has the largest Raman enhancement for the tested molecule suggesting their strength and potential on SERS enhancement in plasmonic gold nanoparticles.

\section{Conclusions}

Feasibility of a multiresonant plasmonic platform based on two types of gold nanostars: LPNs with $20 \mathrm{~nm}$ radius and SPNs with $2 \mathrm{~nm}$ radius have been demonstrated. The proposed design offers a high SERS intensity enhancement of $85 \%$ compared with the LPNs plasmonic platforms. The improvement of the platform sensitivity is related to the refractive index of solvents. The proposed platform has the combined effect of SPNs and LPNs which might tune the sensitivity of sensors in the near infrared regions. These findings may pave the way for the manufacturing of a new type of plasmonic platforms based on a heterogeneous shape of the nanostructures for the detection at the molecular level.

\section{Acknowledgment}

C. Dab is grateful for an FRQNT (Fonds
Québécois de la Recherche sur la Nature et les Technologies) postdoctoral scholarship. A. Ruediger acknowledges generous support through CMC microsystems and an NSERC discovery grant.

Open Access This article is distributed under the terms of the Creative Commons Attribution 4.0 International License (http://creativecommons.org/licenses/by/4.0/), which permits unrestricted use, distribution, and reproduction in any medium, provided you give appropriate credit to the original author(s) and the source, provide a link to the Creative Commons license, and indicate if changes were made.

\section{References}

[1] G. Demirel, H. Usta, M. Yilmaz, M. Celik, H. Ardıç Alidağı, and F. Buyukserin, "Surface-enhanced Raman spectroscopy (SERS): an adventure from plasmonic metals to organic semiconductors as SERS platforms," Journal of Materials Chemistry C, 2018, 6(20): 5314-5335.

[2] G. V. Maltzahn, A. Centrone, J. H. Park, R. Ramanathan, M. J. Sailor, T. Alan Hatton, et al., "SERS-coded gold nanorods as a multifunctional platform for densely multiplexed near-infrared imaging and photothermal heating," Advanced Materials, 2009, 21(31): 3175-3180.

[3] F. H. L. Koppens, D. E. Chang, and F. J. García De Abajo, "Graphene plasmonics: a platform for strong light-matter interactions," Nano Letters, 2011, 11(8): 3370-3377.

[4] D. Chahinez, T. Reji, and R. Andreas, "Modeling of the surface plasmon resonance tunability of silver/gold core-shell nanostructures," RSC Advances, 2018, 8(35): 19616-19626.

[5] T. Wei, Y. Liu, W. Dong, Y. Zhang, C. Huang, Y. Sun, et al., "Surface-dependent localized surface plasmon resonances in CuS nanodisks," ACS Applied Materials \& Interfaces, 2013, 5(21): 10473-10477.

[6] L. J. Sherry, S. H. Chang, G. C. Schatz, R. P. Van Duyne, B. J. Wiley, and Y. Xia, "Localized surface plasmon resonance spectroscopy of single silver nanocubes," Nano Letters, 2005, 5(10): 2034-2038.

[7] K. Kneipp, Y. Wang, H. Kneipp, L. T. Perelman, I. Itzkan, R. R. Dasari, et al., "Single molecule detection using surface-enhanced Raman scattering (SERS)," Physical Review Letters, 1997, 78(9): 1667-1670.

[8] K. Kneipp, H. Kneipp, V. Kartha, R. Manoharan, G. Deinum, I. Itzkan, et al., "Detection and identification of a single DNA base molecule using surface-enhanced Raman scattering (SERS)," Physical Review E, 1998, 57(6): 6281-6284. 
[9] A. M. Michaels, J. Jiang, and L. Brus, "Ag nanocrystal junctions as the site for surface-enhanced Raman scattering of single rhodamine 6G molecules," The Journal of Physical Chemistry B, 2000, 104(50): 11965-11971.

[10] P. Strobbia, E. Languirand, and B. M. Cullum, "Recent advances in plasmonic nanostructures for sensing: a review," Optical Engineering, 2015, 54(10): 100902.

[11] K. M. Mayer and J. H. Hafner, "Localized surface plasmon resonance sensors," Chemical Reviews, 2011, 111(6): 3828-3857.

[12] E. Ozbay, "Plasmonics: merging photonics and electronics at nanoscale dimensions," Science, 2006, 311(5758): 189-193.

[13] P. K. Jain, X. H. Huang, I. H. El-Sayed, and M. A. El-Sayed, "Noble metals on the nanoscale: optical and photothermal properties and some applications in imaging, sensing, biology, and medicine," Accounts of Chemical Research, 2008, 41(12): $1578-1586$.

[14] P. C. Ray, "Size and shape dependent second order nonlinear optical properties of nanomaterials and their application in biological and chemical sensing," Chemical Reviews, 2010, 110(9): 5332-5365.

[15] M. Tabatabaei, A. Sangar, N. Kazemi-Zanjani, P. Torchio, A. Merlen, and F. Lagugné-Labarthet, "Optical properties of silver and gold tetrahedral nanopyramid arrays prepared by nanosphere lithography," The Journal of Physical Chemistry C, 2013, 117(28): 14778-14786.

[16] H. Yuan, A. M. Fales, C. G. Khoury, J. Liu, and T. Vo-Dinh, "Spectral characterization and intracellular detection of surface-enhanced Raman scattering (SERS)-encoded plasmonic gold nanostars," Journal of Raman Spectroscopy, 2013, 44(2): 234-239.

[17] J. Kumar, R. Thomas, R. S. Swathi, and K. G. Thomas, "Au nanorod quartets and Raman signal enhancement: towards the design of plasmonic platforms," Nanoscale, 2014, 6(18): 10454-10459.

[18] A. Garreau, M. Tabatabaei, R. Hou, G. Q. Wallace, P. R. Norton, and F. Lagugné-Labarthet, "Probing the plasmonic properties of heterometallic nanoprisms with near-field fluorescence microscopy," The Journal of Physical Chemistry C, 2016, 120(36): 20267-20276.

[19] G. Q. Wallace, M. Tabatabaei, R. Hou, M. J. Coady, P. R. Norton, T. S. Simpson, et al., "Superimposed arrays of nanoprisms for multispectral molecular plasmonics," ACS Photonics, 2016, 3(9): $1723-1732$.

[20] C. Dab, C. Awada, A. Merlen, and A. Ruediger, "Near-field chemical mapping of gold nanostructures using a functionalized scanning probe," Physical Chemistry Chemical Physics, 2017, 19(46): 31063-31071.

[21] W. Zhang, Q. Li, and M. Qiu, "A plasmon ruler based on nanoscale photothermal effect," Optics Express, 2013, 21(1): 172-181.

[22] J. B. Lassiter, J. Aizpurua, L. I. Hernandez, D. W. Brandl, I. Romero, and S. Lal, "Close encounters between two nanoshells," Nano Letters, 2008, 8(4): 1212-1218.

[23] R. Esteban, A. G. Borisov, P. Nordlander, and J. Aizpurua, "Bridging quantum and classical plasmonics with a quantum-corrected model," Nature Communications, 2012, 3: 825-829.

[24] Q. Yan, A. Chen, S. J. Chua, and X. S. Zhao, "Nanosphere lithography from template-directed colloidal sphere assemblies," Journal of Nanoscience and Nanotechnology, 2006, 6(6): 1815-1818.

[25] G. H. Chan, J. Zhao, E. M. Hicks, G. C. Schatz, and R. P. Van Duyne, "Plasmonic properties of copper nanoparticles fabricated by nanosphere lithography," Nano Letters, 2007, 7(7): 1947-1952.

[26] C. L. Haynes and R. P. Van Duyne, "Dichroic optical properties of extended nanostructures fabricated using angle-resolved nanosphere lithography," Nano Letters, 2003, 3(7): 939-943.

[27] G. Kolhatkar, A. Merlen, J. Zhang, C. Dab, G. Q. Wallace, F. Lagugné-Labarthet, et al., "Optical near-field mapping of plasmonic nanostructures prepared by nanosphere lithography," Beilstein Journal of Nanotechnology, 2018, 9(1): 1536-1543.

[28] N. F. Van Hulst, "Nanophotonics: plasmon quantum limit exposed," Nature Nanotechnology, 2012, 7(12): 775-7.

[29] S. K. Ghosh, S. Nath, S. Kundu, K. Esumi, and T. Pal, "Solvent and ligand effects on the localized surface plasmon resonance (LSPR) of gold colloids," The Journal of Physical Chemistry B, 2004, 108(37): 13963-13971.

[30] R. Morarescu, H. Shen, R. A. L. Vallée, B. Maes, B. Kolaric, and P. Damman, "Exploiting the localized surface plasmon modes in gold triangular nanoparticles for sensing applications," Journal of Materials Chemistry, 2012, 22(23): 11537-11542.

[31] C. Dab, R. Thomas, and A. Ruediger, "Modeling of the surface plasmon resonance tunability of silver/gold core-shell nanostructures," RSC Advances, 2018, 8(35): 19616-19626.

[32] G. Kolhatkar, J. Plathier, and A. Ruediger, "Nanoscale investigation of materials, chemical reactions, and biological systems by tip enhanced Raman spectroscopy - a review," Journal of Materials Chemistry C, 2018, 6(6): 1307-1319.

[33] E. Karimi, S. A. Schulz, I. De Leon, H. Qassim, J. Upham, and R. W. Boyd, "Generating optical orbital angular momentum at visible wavelengths using a plasmonic metasurface," Light: Science \& Applications, 2014, 3(5): 1-4.

[34] A. Tittl, P. Mai, R. Taubert, D. Dregely, N. Liu, and H. Giessen, "Palladium-based plasmonic perfect 
absorber in the visible wavelength range and its application to hydrogen sensing," Nano Letters, 2011, 11(10): 4366-4369.

[35] X. Chen, L. Huang, H. Mühlenbernd, G. Li, B. Bai, Q. Tan, et al., "Dual-polarity plasmonic metalens for visible light," Nature Communications, 2012, 3: 1-6.

[36] H. Liao, C. L. Nehl, and J. H. Hafner, "Biomedical applications of plasmon resonant metal nanoparticles," Nanomedecine, 2006, 1(2): 201-208.

[37] A. M. Smith, M. C. Mancini, and S. Nie, "Bioimaging: second window for in vivo imaging," Nature Nanotechnology, 2009, 4(11): 710-711.

[38] L. Qin, S. Zou, C. Xue, A. Atkinson, G. C. Schatz, and C. A. Mirkin, "Designing, fabricating, and imaging Raman hot spots," Proceedings of the National Academy of Sciences, 2006, 103(36):
13300-13303.

[39] J. P. Camden, J. A. Dieringer, J. Zhao, R. P. Van Duyne, and R. P. V. A. N. Duyne, "Controlled plasmonic nanostructures for surface-enhanced spectroscopy and sensing," Accounts of Chemical Research, 2008, 41(12): 1653-1661.

[40] Y. Chen, K. Munechika, and D. S. Ginger, "Dependence of fluorescence intensity on the spectral overlap between fluorophores and plasmon resonant single silver nanoparticles," Nano Letters, 2007, 7(3): 690-696.

[41] F. S. Ou, M. Hu, I. Naumov, A. Kim, W. Wu, A. M. Bratkovsky, et al., "Hot-spot engineering in polygonal nanofinger assemblies for surface enhanced Raman spectroscopy," Nano Letters, 2011, 11(6): 2538-2542. 